Internist $2011 \cdot 52: 1275-1275$

DOI 10.1007/s00108-011-2833-4

Online publiziert: 16. Oktober 2011

(c) Springer-Verlag 2011

\author{
B.E. Strauer ${ }^{1} \cdot$ S.M. Schellong ${ }^{2}$ \\ ${ }^{1}$ Heinrich-Heine-Universität Düsseldorf \\ ${ }^{2}$ Medizinische Klinik 2, Städtisches Krankenhaus Dresden-Friedrichstadt, Dresden
}

\title{
Antithrombotische Behandlungsstrategien
}

prophylaktisch anzugehen, es rechtzeitig zu erkennen und zu behandeln.

Behandlungsstrategien ergibt sich aus der hohen Krankheitspotenz thromboembolischer Erkrankungen: Allein in Europa wird die Rate an thromboembolischen Erkrankungen auf ca. 6 Mio. geschätzt. Alle Fächer der Medizin, konservativ oder operativ, kommen damit in Berührung. Die venöse Thrombose weist eine Inzidenz von ca. 2:100o pro Jahr auf, mit deutlicher Altersabhängigkeit bei über 45-Jährigen. Oft sind erstmals entstandene Thrombosen Ausdruck eines Malignoms. Rund 2/3 aller Thrombosen treten in Zusammenhang mit zeitlich begrenzten Risikosituationen auf, z. B. perioperativ. Etwa 30.00o Patienten versterben pro Jahr an den Folgen einer Lungenembolie, deren zugrunde liegende Thrombose lediglich in weniger als $1 / 3$ der Patienten intra vitam diagnostiziert wird.

Wichtige Manifestationen thromboembolischer Erkrankungen (venös, arteriell) sind das akute Koronarsyndrom, der akute Herzinfarkt, der Schlaganfall, die tiefe Beinvenenthrombose, die Lungenembolie und die periphere arterielle Verschlusskrankheit. Dabei zeigen prädisponierende Faktoren, wie Vorhofflimmern, Herzklappenerkrankungen und Herzinsuffizienz eine hohe Krankheitswertigkeit.

Zur Behandlung dieser Erkrankungen kommen entsprechend der traditionellen Einteilung Thrombozytenaggregationshemmer, Antikoagulanzien und Thrombolytika in Betracht. Diese 3 Substanzgruppen sind stets unter strenger Abwägung möglicher Risiken und Komplikationen einzusetzen. Insbesondere gilt es, ein mögliches therapieinduzierte Auftreten intestinaler und zerebraler Blutungen

\section{- Die Vermeidung von Blutungs- komplikationen ist beim Einsatz antithrombotischer Therapien vorrangig.}

Das bislang für die Therapie mit Antikoagulanzien (z. B. Vitamin-K-Antagonisten) obligate und für die Vielzahl der behandelten Patienten oft beschwerliche Gerinnungsmonitoring (Quick-Wert, International Normalized Ratio) kann möglicherweise künftig bei Verwendung neuerer Substanzen reduziert bzw. aufgehoben werden. Diese oralen Thrombininhibitoren werden nicht über das $\mathrm{P}_{450}$ System metabolisiert, haben keinen genetischen Polymorphismus und erfordern keine ständige Dosisanpassung. Neuere Studien (Rocket AF, Einstein-DVT, RELY u. a.) haben gezeigt, dass z. B. der oral verwendbare Thrombin- (Faktor II-) Inhibitor Dabigatran sowie der Faktor-XaAntagonist Rivaroxaban bei der Behandlung des Vorhofflimmerns Vorteile bieten. Erfreulicherweise könnte auch bei Verwendung der neuen Inhibitoren die überlappende, perioperative und oft zeitaufwendige Heparintherapie bei Übergang von Vitamin-K-Antagonisten auf Heparin und umgekehrt verkürzt werden. Nach vollständiger Auswertung der laufenden Studien wird sich zeigen, ob die „Marcumar-Ära“ zu Gunsten der sog. „Neuen Antikoagulanzien“ beendet werden kann.

\footnotetext{
- Durch die Neuentwicklung oraler Thrombininhibitoren hat ein Umdenken in der oralen Langzeitantikoagulation begonnen.
}

Das vorliegende Heft zum Schwerpunktthema der „antithrombotischen Strategien" fokussiert die angesprochenen Krankheitsbilder und zeigt neue Therapiemöglichkeiten, insbesondere unter dem Blickwinkel der neuen antithrombotischen Substanzentwicklungen.

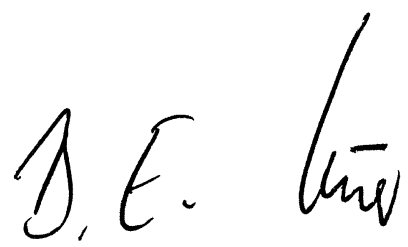

B.E. Strauer

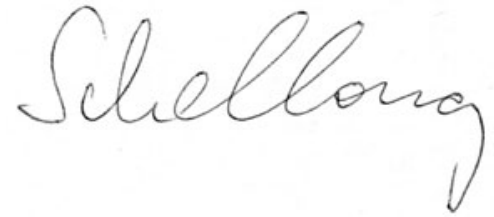

S.M. Schellong

\section{Korrespondenzadressen}

Prof. Dr. B.E. Strauer

Heinrich-Heine-Universität Düsseldorf

Moorenstraße 5, 40225 Düsseldorf

strauer@med.uni-duesseldorf.de

Prof. Dr. S.M. Schellong

Medizinische Klinik 2, Städtisches Krankenhaus Dresden-Friedrichstadt Friedrichstraße 41, 01067 Dresden schellong-se@khdf.de 